\title{
Clinical and Radiological Comparison of Posterolateral Fusion and Posterior Interbody Fusion Techniques for Multilevel Lumbar Spinal Stabilization In Manual Workers
}

\author{
Hayati Aygün ${ }^{1}$, Albert Çakar ${ }^{1}$, Nergiz Hüseyinoğlu ${ }^{2}$, Urfettin Hüseyinoğlu ${ }^{3}$, Recep Çelik ${ }^{4}$ \\ ${ }^{I}$ Depertment of Orthopaedics, Kafkas Univeristy Medical School, Kars, Turkey \\ ${ }^{2}$ Department of Neurology, Kafkas Univeristy Medical School, Kars, Turkey \\ ${ }^{3}$ Department of Anesthesiology, Kafkas Univeristy Medical School, Turkey \\ ${ }^{4}$ Department of Orthopaedics, Spatial Şifa Hospital, Erzurum, Turkey
}

\begin{abstract}
Study Design: Eighty-four patients who had been treated for degenerative spinal diseases between January 2006 and June 2009 were reviewed retrospectively.

Purpose: We aimed to compare the clinical and radiologic findings of manual workers who underwent posterolateral fusion (PLF) or posterior interbody fusion (PLIF) involving fusion of 3 or more levels of the spine.

Overview of Literature: Previous studies have concluded that there is no significant difference between the clinical outcome of PLF and PLIF techniques.

Methods: After standard decompression, 42 patients underwent PLF and the other 42 patients underwent PLIF. Radiologic findings, Oswestry disability index (ODI) scores, and visual analogue scale (VAS) scores were assessed preoperatively and at 6-month intervals postoperatively and return to work times/rates were assessed for 48 months.

Results: Patients who underwent PLF had significantly shorter surgical time and less blood loss. According to the 48-month clinical results, ODI and VAS scores were reduced significantly in the two groups, but the PLIF group showed better results than the PLF group at the last follow-up. Return to work rate was $63 \%$ in the PLF group and $87 \%$ in the PLIF group. Union rates were found to be $81 \%$ and $89 \%$, respectively, after 24 months $(p=0.154)$.

Conclusions: PLIF is a preferable technique with respect to stability and correction, but the result does not depend on only the fusion rates. Discectomy and fusion mass localization should be considered for achieving clinical success with the fusion technique. Before performing PLIF, the association of the long operative time and high blood loss with mortality and morbidity should be taken into consideration, particularly in the elderly and disabled patients.
\end{abstract}

Keywords: Degenerative spine; Posterolateral fusion; Posterior interbody fusion; Disc disease; Scoliosis; Spinal instability

\section{Introduction}

The prevalence of degenerative spinal diseases, including spinal stenosis, degenerative disk diseases, and degenerative deformities [1] is increasing along with an increase in life expectancy [2]. Manual workers or labourers are

Received Nov 17, 2013; Revised Jan 19, 2014; Accepted Feb 6, 2014

Corresponding author: Hayati Aygün

Department of Orthopaedics, University of Kafkas School of Medicine, Pasacayiri 36000 Kars, Turkey

Tel: +90-532-694-5552, Fax: +90-442-329-0427 , E-mail: hayatiaygun@gmail.com 
Table 1. Occupations of patients

\begin{tabular}{lcc} 
Patients & Posterolateral fusion & Posterior interbody fusion \\
Constuction worker & 12 & 14 \\
Farmer & 14 & 16 \\
\hline House servant & 3 & 3 \\
Waiting woman & 2 & 3 \\
\hline Streetcleaner & 3 & 2 \\
Mechanic & 5 & 3 \\
House painter & 3 & 1 \\
Total & 42 & 42
\end{tabular}

usually employed in many of the industrial fields to perform physical, manual work that includes construction work, farming, house cleaning etc. Owing to the nature of the work, degenerative spine disease is common in this population [3].

Debate continues regarding the treatment of these diseases. While many patients are treated successfully with conservative treatment methods, some patients do not benefit from these treatments. Recently, a consensus has started to form in favor of performing certain surgical procedures in patients who do not respond well to conservative therapies $[1,4]$. However, there is still no agreement on the efficacy of specific surgical procedures.

Posterolateral fusion (PLF) and posterior interbody fusion (PLIF) are the important techniques that are frequently used for achieving fusion during the surgical treatment of instability due to degenerative spine diseases $[5,6]$. While many researchers claim that neither of these techniques is superior to the other clinically, some researchers claim that PLIF provides better biomechanical stability and maintenance of stability. However, in many of these studies, the results of long and short segment fusion were studied together or the results of only short segment fusion were assessed $[5,6]$. Because of the biomechanical differences between the fusion of long and short segments $[7,8]$, we believe that a clinical comparison of these fusion techniques should assess long and short segments separately. On the other hand, in manual laborers the surgical stabilization technique should ensure that they can continue with their jobs. Degenerative spine diseases and instabilities are common in manual laborers and heavy workers [3]. Special importance should be paid to the treatment of these patients with respect of instability.
Previous studies have evaluated the results of long and short segment fusion together. We did not find any previous study that evaluated the results of long segment spinal fusion in manual workers. In this study, a clinical and radiologic comparison between PLF and PLIF was made in manual workers who underwent fusion of 3 or more levels of the spine.

\section{Materials and Methods}

\section{Surgical indications}

The study cohort included manual workers who had been operated for spinal instability, spondylolisthesis, and de novo scoliosis with neurologic symptoms and involving multiple levels of the lumbar region. Patients who had severe osteoporosis (T score -2.5 or lower), infection, or malignancy, and those who could not return to their work after the surgery were not included in the study.

\section{Patients}

A total of 103 manual workers who had undergone surgery between January 2006 and June 2009 were investigated retrospectively. Of these, 84 patients who had regular preoperative and postoperative data and met the pre-specified criteria were selected for this study (Tables $1,2)$. The mean age of the patients in the PLF group was 57.2 years (range, $44-74$ years), and the mean age of the patients in the PLIF group was 56.6 years (range, 47-69 years) (Table 3 ). The study subjects were assembled in a manner that dissociates the exposure from the outcome. Neither physicians helping with the recruitment nor eligible volunteers were aware of the study hypothesis. The 
Table 2. Patient conditions in each surgical technique group

\begin{tabular}{lcc} 
Conditions & Posterolateral fusion & Posterior interbody fusion \\
\hline Spinal stenosis & 4 & 3 \\
\hline Post laminectomy instability & 4 & 7 \\
Spondylolisthesis & 5 & 4 \\
Degenerative disc disease (multilevel) & 9 & 5 \\
Degenerative scoliosis & 20 & 23 \\
Total & 42 & 42 \\
\hline
\end{tabular}

Table 3. Patient characteristics and operative data in both groups

\begin{tabular}{|c|c|c|c|}
\hline Patients & Posterolateral fusion & Posterior interbody fusion & $p$-value \\
\hline Age (yr) & 54.2 & 56.6 & 0.913 \\
\hline Famale & 18 & 16 & 0.647 \\
\hline Male & 24 & 26 & 0.663 \\
\hline \multicolumn{4}{|l|}{ Body mass index } \\
\hline Female & $25.8 \pm 2.4$ & $26.4 \pm 1.4$ & 0.597 \\
\hline Male & $23.4 \pm 2.2$ & $24.3 \pm 3.1$ & 0.651 \\
\hline Operation time (min) & $201 \pm 107.6$ & $309 \pm 103.2$ & 0.019 \\
\hline Blood loss (mL) & $548.2 \pm 216.5$ & $913 \pm 316.5$ & 0.021 \\
\hline Average fused segments & $4.1 \pm 1.07$ & $3.6 \pm 1.40$ & 0.048 \\
\hline
\end{tabular}

study design was approved by the Institutional Ethics Review Board (IRB No: 80576354-050-99/55).

\section{Surgical treatment}

Spinal cord and nerve root decompression (laminectomy, hypertrophic ligamentum flavum excision, and facetectomy) was performed as needed in all of the patients who underwent surgical treatment. Discectomy was performed in all patients of the PLIF group and in patients of the PLF group if needed. In the 42 patients who were included in the PLF group, titanium polyaxial pedicular screws (Legacy, Sofamor Danek, Memphis, TN, USA) and autografts with demineralized bone matrix (DBM) (Johnson \& Johnson, Raritan, NJ, USA) were used to stabilize the spine. The mixture was placed after the transverse processes were decorticated (PLF). The 42 patients who were included in the PLIF group, titanium polyaxial pedicular screws (Legacy, Medtronic, Sofamor Danek), and the autograft+DBM mixture were placed after discectomy was performed from the posterior and with expandable interbody cage (B twin, Disc O Tech, Tel Aviv, Israel) (PLIF).

Fusion of three or more levels of the spine was performed (3-6 levels). The uppermost fusion level was the 11th thoracic vertebra (Th11) and the lowermost fusion level was the first sacral vertebra (S1).

\section{Assessment of outcomes}

Patient outcomes were assessed based on the patient data obtained from hospital records, using the visual analogue scale (VAS) and Oswestry disability index (ODI) before the surgery and at every 6 months thereafter for 48 months. Blood loss and surgical time were evaluated in both the groups. Lumbar, sagittal, and coronal angles were measured on the images obtained in the standing position both preoperatively and postoperatively. Lumbar lordosis (sagittal plane) and lumbar scoliosis (coronal plane) were evaluated according to the Cobb method. Lumbar lordosis was identified on the basis of the S1 inferior endplate and lumbar 1 (L1) superior endplate, 
and deformity was identified on the basis of the superior endplate of the deformity apex and the inferior endplate of the caudal part of the deformity in the coronal plane.

The anteroposterior and lateral X-ray radiograms and computed tomography (CT) scans were performed for assessing the fusion. In both groups, the subjects who had not undergone a CT scan during the follow-up for assessing the fusion were asked to undergo a thin-cut CT scan. Two and half millimeter axial CT sections were obtained through the fusion mass as well as the adjacent segments with $1.25 \mathrm{~mm}$ reconstructions and both sagittal and curved coronal reformats.

According to the Lenke method, the four fusion grades as judged from the anteroposterior radiograph were as follows: grade A is definitely solid with bilateral stout fusion masses present; grade B is probably solid with a unilateral stout fusion mass and contralateral thin fusion mass; grade $\mathrm{C}$ is probably not solid with a thin unilateral fusion mass; and grade $\mathrm{D}$ is definitely not solid with thin fusion masses bilaterally with obvious pseudoarthrosis or bone graft dissolution bilaterally.

Interbody fusion was graded by the method of Brantigan and Steffee as modifed to describe the Fraser defnition of locked pseudarthrosis (BSF scale). BSF-1: radiographical pseudarthrosis is indicated by collapse of the construct, loss of disc height, vertebral slip, displacement of the carbon cage, broken screws, signicant resorption of the bone graft, or lucency visible around the periphery of the graft or cage. BSF-2: radiographical locked pseudarthrosis is indicated by lucency visible in the middle of the cages with solid bone growing into the cage from each vertebral endplate. BSF-3: radiographical fusion: bone bridges over at least half of the fusion area with at least the density originally achieved at surgery. Radiographical fusion through one cage (half of the fusion area) is considered to be mechanically solid fusion even if there is lucency on the opposite side [9].

To minimize any possible errors regarding the interpretation of X-rays, all graphies were evaluated by another orthopedist and radiologist, in addition to evaluation by the operating team.

Results were analyzed statistically using SPSS ver. 15.0 (SPSS Inc., Chicago, IL, USA).

Descriptive statistics, including frequency and percentage, were determined. All data sets were tested for normal distribution using the Kolmogorov-Smirnov test. Measurements were compared between groups using the unpaired Student's $t$ test or the Mann-Whitney $U$ test for normally and non-normally distributed variables, respectively. $p<0.05$ was considered statistically significant.

\section{Results}

Clinical and radiologic outcomes were evaluated for 48 months retrospectively in each cohort. The mean surgical time was significantly lower in the PLF group than in the PLIF group $(201 \pm 107.6 \mathrm{~min}$ vs. $309 \pm 103.2 \mathrm{~min}$, respectively; $p=0.019$ ) (Table 2). Blood loss in the PLF group $(548.2 \pm 216.5 \mathrm{~mL})$ was significantly less than that in the PLIF group $(913 \pm 316.5 \mathrm{~mL})(p=0.021)$ (Table 3$)$.

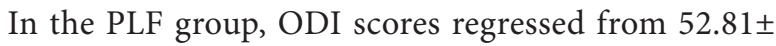
9.09 (preoperatively) to $11.98 \pm 3.10$ (postoperatively). In the PLIF group, ODI scores regressed from $49.73 \pm 4.13$ (preoperatively) to $7.84 \pm 4.02$ (postoperatively). This difference between the PLF and PLIF groups was significant ( $p=0.014$ ) (Table 4). Comparison of lumbar and lower limb VAS scores between the preoperative and postoperative periods showed that while the VAS score regressed from $7.91 \pm 1.34$ to $4.62 \pm 1.98$ in the PLF group, it regressed from $8.18 \pm 0.98$ to $3.24 \pm 1.14$ in the PLIF group $(p=0.000)$ (Table 5). The mean return to work time (MRWT) was 9 weeks (range, 3-15 weeks) in the PLF group and 6 weeks (range, 3-11 weeks) in the PLIF group, postoperatively. During the pre-specified time interval, the return to work rates (RWR) were found to be $63 \%$ in the PLF group and $87 \%$ in the PLIF group ( $p=0.012$ ) (Table 6). Lumbar, sagittal, and coronal angle images from patients in both the groups were compared radiologically. In the PLF group, the mean preoperative coronal plane lumbar angle $\left(11.4^{\circ}\right)$ decreased to $5.2^{\circ}$ postoperatively $(p=0.023)$. In the PLIF group, this angle decreased from $16.2^{\circ}$ preoperatively to $4.2^{\circ}$ postoperatively $(p=0.000)$. The lumbar lordosis angles increased from $22.4^{\circ}$ preoperatively to $27.5^{\circ}$ postoperatively in the PLF group ( $p=0.026$ ) and from $20.7^{\circ}$ preoperatively to $35.3^{\circ}$ postoperatively in the PLIF group $(p=0.011)$ (Table 7).

Union rates were found to be 55\% in the PLF group and $62 \%$ in the PLIF group after 6 months and $81 \%$ and $89 \%$, respectively, after 24 months ( $p=0.154$ ).

Comparison of complications between the 2 groups showed that while dural tears and radiculopathy were common in the PLIF group, disc space loss and screw pull-out were more frequent in the PLF group $(p=0.000)$ 
Table 4. Preoperative and postoperative pain assessment by Oswestry disability index

\begin{tabular}{lccc} 
Oswestry disability index & Posterolateral fusion & Posterior interbody fusion & $p$-value \\
Preoperative & $52.81 \pm 9.09$ & $49.73 \pm 4.13$ & 0.746 \\
\hline Postoperative & & & 0.938 \\
\hline 6 mo & $31.94 \pm 4.39$ & $32.01 \pm 6.10$ & 0.954 \\
\hline 9 mo & $29.28 \pm 2.87$ & $29.33 \pm 5.29$ & 0.045 \\
\hline 12 mo & $20.19 \pm 5.08$ & $18.24 \pm 2.61$ & 0.053 \\
\hline 18 mo & $16.67 \pm 3.61$ & $14.72 \pm 2.14$ & 0.048 \\
\hline 24 mo & $15.44 \pm 2.57$ & $12.49 \pm 3.68$ & 0.043 \\
\hline 30 mo & $14.53 \pm 6.33$ & $9.78 \pm 5.58$ & 0.039 \\
\hline 36 mo & $12.55 \pm 5.67$ & $7.72 \pm 2.15$ & $7.81 \pm 1.24$ \\
\hline 42 mo & $12.01 \pm 2.11$ & $7.84 \pm 4.02$ & 0.031 \\
\hline 48 mo & $11.98 \pm 3.10$ & & 0.036 \\
\hline
\end{tabular}

Table 5. Preoperative and postoperative pain assessment by visual analogue scale

\begin{tabular}{lccc} 
Visual analogue scale & Posterolateral fusion & Posterior interbody fusion & $p$-value \\
\hline Preoperative & $7.91 \pm 1.34$ & $8.18 \pm 0.98$ & 0.068 \\
\hline Postoperative & & & 0,053 \\
\hline 6 mo & $5.64 \pm 0.68$ & $5.21 \pm 1.36$ & 0.035 \\
\hline 12 mo & $5.68 \pm 1.12$ & $4.03 \pm 0.73$ & 0.037 \\
\hline 18 mo & $5.36 \pm 0.91$ & $4.00 \pm 1.19$ & 0.033 \\
\hline 24 mo & $4.75 \pm 1.82$ & $4.01 \pm 0.22$ & 0.024 \\
\hline 30 mo & $4.68 \pm 1.33$ & $3.19 \pm 0.62$ & 0.029 \\
\hline 36 mo & $4.59 \pm 0.76$ & $3.24 \pm 1.21$ & 0.027 \\
\hline 42 mo & $4.50 \pm 1.11$ & $3.22 \pm 0,21$ & 0.025 \\
\hline 48 mo & $4.62 \pm 1.98$ & $3.24 \pm 1.14$ & \\
\hline
\end{tabular}

Table 6. Return to work times and rates

\begin{tabular}{lcccc} 
Patients & Posterolateral fusion & Posterior interbody fusion & $p$-value \\
Mean return to work time (wk) & 9 & 6 & 0.004 \\
Rate of returnt to work (\%) & 63 & 87 & 0.012 \\
\hline
\end{tabular}

Table 7. Radiographic assessment of lumbar alignment

\begin{tabular}{|c|c|c|c|c|c|c|}
\hline \multirow{2}{*}{$\begin{array}{l}\text { Radiological angles } \\
\text { (degree) }\end{array}$} & \multicolumn{3}{|c|}{ Posterolateral fusion } & \multicolumn{3}{|c|}{ Posterior interbody fusion } \\
\hline & Preoperative & Postoperative & $p$-value & Preoperative & Postoperative & $p$-value \\
\hline Coronal angles & 11.4 & 5.12 & 0.023 & 16.2 & 4.2 & 0.000 \\
\hline Lomber lordosis angle & 22.4 & 27.5 & 0.026 & 20.7 & 35.3 & 0.011 \\
\hline
\end{tabular}


Table 8. Complications in each surgical technique group

\begin{tabular}{lccc} 
Complications & Posterolateral fusion & Posterior interbody fusion & $p$-value \\
Radiculopathy & 2 & 5 & 0.004 \\
\hline Displacement of spacer & 0 & 1 & 0.000 \\
\hline Dural tear & 1 & 3 & 0.001 \\
\hline Degenerative adjacent disc & 7 & 8 & 0.086 \\
\hline Failure of hardware & 3 & 1 & 0.000 \\
Lost of disc space & 7 & 1 & 0.003 \\
Infection & 2 & 0 & 0.045 \\
Pseudoarthrosis & 3 & 19 & 0.000 \\
\hline Total & 25 & 0.042 & 0.000 \\
\hline
\end{tabular}

PLF, posterolateral fusion; PLIF, posterior interbody fusion.

(Table 8). The dura mater was repaired using dura graft (Duraform, Codman \& Shurtleff Inc., Raynham, MA, USA) in the patient who had experienced a dural tear. One of the patients in the PLF group had experienced severe lower back pain. Evaluation at 24 months revealed loosening of the screws; a revision was performed, and this patient was excluded from the study. No additional interventions were required except for physical therapy and support for patients who had a partial screw pull-out.

One of the patients in the PLIF group had experienced cerebrospinal fluid leak? lasting for 3 weeks; this patient was treated by performing wound care and applying conservative bandages. Two patients in the PLF group and 1 patient in the PLIF group had elevated white blood cell count and C-reactive protein levels that persisted for approximately 3 months. Tests for infection had not shown any bacteria in the cultures. All patients were treated with empirical antibiotic therapy (sodium fucidate, $1.5 \mathrm{gr}$ daily).

\section{Discussion}

There is no uniform agreement among the surgeons on the indications for spinal fusion. No generally accepted criteria have been developed on this issue. Many criteria described in the literature appear to permissive and subjective: prolapsed disc in young individuals who wish to return to the same type of manual work, disc disease with disc space narrowing, disc herniation with a longstanding history of low back pain, low back pain being greater than leg pain etc. [10]. Although these criteria are useful in deciding whether or not to fuse a given motion segment, they do not provide an objective assessment of the source of pain and disability, nor do they ensure that the patients will actually benefit from the arthrodesis.

According to Kambin [10] clinically, the surgical stabilization of a motion segment is justified under the following set of circumstances: 1) When the ability of the intervertebral disc to contain and transmit the external forces has been altered. For instance, localized degenerative disc pathology as well as degenerative spondylolisthesis and retrolisthesis associated with radiographic evidence of hypermobility on lateral flexion and extension or anteroposterior side-bending films. In addition, symptom producing adult scoliotic curves fall in this category. 2) Some conditions in which the integrity of the structures of the motion segments has been compromised, such as developmental instabilities associated with a defect in the pars inter-articularis; surgically induced instability; and post-traumatic fractures or ligamentous injuries; infections and osteomyelitis.

Generally there are three requisites for surgical stabilization of spinal units; positive provocative and anesthetic testing and abnormal dynamic studies.

Multilevel fusions are rarely required for the treatment of degenerative spinal diseases and are generally avoided due to the risk of adjacent segment disease and the restriction of intervertebral movement. Relieving symptoms and achieving a permanent cure is thus difficult in disc diseases that involve degeneration at multiple levels of the spine. Effective and sufficient stabilization is very important for preventing iatrogenic instability during the treatment of multilevel severe spinal osteoarthritis, severe spinal stenosis, grade 3 or 4 spondylolisthesis, degenera- 
tive lumbar scoliosis, and revision surgery for multilevel disc diseases. To meet this need, many fusion techniques have been developed for achieving spinal stabilization $[11,12]$. Alignment disorders and instability are considered to be the major factors influencing the development of degenerative spinal diseases [13]. Degeneration of biological structures (bone, cartilage, and ligaments) due to aging, lifestyle, and genetic characteristics of the individual result in a diminished ability of the spine to carry weight $[14,15]$. Thus, spinal cord and root compression occurs as a result of disc space narrowing, impairment of coronal and sagittal balance, increased instability-related osteophytic changes, and hypertrophic changes in the tendons. The term segmental instability has not been well defined in the literature. However, it is commonly used as an indication for surgical fusion of the spinal unit. White and Panjabi [16] defined clinical instability as loss of the ability of the spine under physiologic loads to maintain relationships between vertebrae in such a way that there is neither damage nor subsequent irritation to the spinal cord or nerve roots. Frymoyer and Selby [17] classified various instabilities as axial, rotational, translational, retrolisthetic, and postsurgical.

The aim of the treatment for such diseases is the elimination of biomechanical insufficiencies and degenerative changes. Thus, successful treatment must include both the elimination of spinal cord and root compression (decompression) and sustainable stabilization that will adapt to life conditions. While some of the techniques provide stabilization from the posterior side only (PLF), the vast majority provide circular fusion (PLIF, anterior lumbar interbody fusion, transforaminal lumbar interbody fusion, extreme lumbar interbody fusion). The aim of these circular fusion techniques is to provide a more stable spinal segment $[11,12]$.

Our study investigated the outcomes of PLF and PLIF in manual workers who had spinal diseases affecting 3 or more levels so as to compare the effectiveness of these techniques in long fusion segments. We observed decreased lumbar lordosis over the long term in multilevel fusions using the PLF technique. However, the improvement in lumbar lordosis was more permanent in patients who underwent PLIF ( $p=0.011$ ). Disc space loss or inadequate restoration of the disc space in patients who underwent PLF may have played a role in causing this difference. The PLIF technique is reported to be advantageous in terms of restoring and maintaining the disc space [18-21]. Our data demonstrate that while PLF had significant benefits over PLIF with respect to the surgical time required and blood loss, PLIF yielded significantly better results as assessed by the ODI, lumbar and lower limb VAS scores, return to work time and median time of return to work, and analysis of lordosis angles. Union rates were found to be similar for the two techniques (PLIF, 89\%; PLF, 81\%; $p=0.154$ ) and correlation was not found between union rates and clinical data.

On the basis of vertebral column biomechanics and axial loading properties, body weight has been shown to be carried by vertebral corpuses and discs [22]. In patients undergoing PLF, the load must be carried by the posterior column instead of the anterior column $[23,24]$. The broad contact surface of the vertebral plates in the lumbar region, its adequate blood supply, and a natural exposure of interbody grafts to compression forces while the patient is ambulatory have contributed to the high success rate and wide acceptance of anterior column stabilization when fusion of lumbar segments is deemed decessary [25]. This may have played an important role in manual workers who overloaded their vertebral columns during their work. Therefore, overloading of the rods and screws before fusion and lateral and posterior bone fusion mass overloading the adjacent screws and rods should be expected [22]. In patients treated with PLIF, the anterior column is supported by interbody cages. This arrangement better supports the natural biomechanics of the spinal column in normal or overloading circumstances [23-25]. We observed a difference between the PLF and PLIF techniques with respect to VAS and ODI score data. While these parameters tended to be fixed in the patients of the PLF group after 18-24 months, they continued to decline in the patients of the PLIF group (Tables 4, 5). Differences in the biomechanics involved in the load-carrying capacity of the fusion segments might have played a role in causing this difference between patients in the PLF and PLIF groups. However, biomechanical studies must be carried out after spinal fusion to determine whether this is indeed the case.

The PLIF technique showed better results in terms of return to work times and rates than the PLF technique (Table 6). This finding suggested that the patients in the PLIF group could return to work earlier than those in the PLF group (mean 6 weeks, 87\%; mean 9 weeks, 63\%, respectively). Fifteen patients in the PLF group and 6 patients in the PLIF group discontinued or changed their 
type of work.

The PLF technique was associated with better radiologic results in the sagittal and coronal planes (Table 7). Although there was a difference between the coronal alignments obtained with the 2 techniques, it was not significant $(p=0.067$ ). Radiologic fusion rates were $81 \%$ in the PLF group and $89 \%$ in the PLIF group ( $p=0.154$ ). Better fusion rates following PLIF may account for the more favorable clinical outcomes in these patients. But, there were little correlation between the fusion rates and clinical data.

On the other hand, some of the patients in the PLF group had continuous low back pain despite the good posterolateral fusion mass. This situation cannot be explained only by biomechanical stability, but discectomy was not performed in these patients. In the literature, discogenic back pain has been reported after PLF procedures in patients despite having a good fusion mass $[16,26,27]$. However in the PLIF group, discectomy was mandatory. It has been speculated that loss of annular integrity and expulsion of nuclear material are associated with local inflammatory response in the epidural space and nerve roots. The addition of mechanical compression on the already inflamed nerve root by the herniated disc is then associated with the onset of symptomatology and the clinical development of pain $[28,29]$. In this study, in some of the PLF patients who had a good fusion mass and spinal alignment without instability, there was continuous low back pain. It is possible that the postoperative back pain can be due ligamentous and muscular problems, but we could not find sufficient information regarding this issue in the literature. This suggests that discectomy may have played another role in the PLIF group, and it showed better results than the PLF group. However, this finding needs to be confirmed in a further study.

Our data indicate that the outcome of PLF technique was better with respect to surgical time and blood loss $(p=0.021)$. This difference most likely indicates that the PLIF technique is more complicated, requiring discectomy and interbody cage placement at multiple spinal levels.

A significant difference was found between PLIF and PLF techniques in terms of postoperative complications. Higher rates of pseudoarthrosis, screw loosening, and disc space loss in patients who underwent PLF account for this difference (Table 8). Dural tear and radiculopathy occurred more often in patients who underwent PLIF (Table 8). PLIF technique showed significantly worse outcomes than PLF technique in terms of intraoperative complications. But, PLF technique showed worse outcomes than PLIF technique in terms of late complications (Table 8). Adjacent segment pathologies (ASP) is a common complication after fusion [30]. In this study, the occurrence of ASP after PLF and PLIF techniques was similar, which is consistent with that reported in the literature.

This study has some limitations. First, this study did not include any other patients than manual workers; the study population lacked homogeneity, it would have been better if this study had included more homogeneous groups. Secondly, discectomy was not performed in some patients of the PLF group who had continuous low back pain despite having a good fusion mass (it was thought to be unnecessary according to the radiographic and clinical assessment performed while making the decision). Thirdly, fusion mass localization or properties of mechanical load sharing may have an important role in these results. In this study, this situation occurred but it could not be completely explained, it would have been more appropriate if the fusion rates and clinical data were assessed and compared separately.

\section{Conclusions}

PLIF technique provides better bone fusion rate and correction of spinal alignment in degenerative spine diseases and deformities as known by spine surgeon, but this study emphasized that clinical results are not dependent on only the fusion rates in manual workers who have to perform heavy work. PLIF and PLF techniques may be preferred for treatment of multilevel degenerative spinal diseases and instabilities in patients who have not responded well to conservative treatments. But if the PLF technique is selected, the importance of discectomy should be taken into account.

Spinal fusion surgery is associated with potential risk of complications; blood loss, infection, and adjacent segment disease. The association of longer surgical time and more blood loss with mortality and morbidity should be taken into consideration while selecting the PLIF technique, particularly in the elderly and severely ill patients.

\section{Conflict of Interest}

No potential conflict of interest relevant to this article 
was reported.

\section{References}

1. Issack PS, Cunningham ME, Pumberger M, Hughes AP, Cammisa FP Jr. Degenerative lumbar spinal stenosis: evaluation and management. J Am Acad Orthop Surg 2012;20:527-35.

2. Atlas SJ, Keller RB, Wu YA, Deyo RA, Singer DE. Long-term outcomes of surgical and nonsurgical management of lumbar spinal stenosis: 8 to 10 year results from the maine lumbar spine study. Spine (Phila Pa 1976) 2005;30:936-43.

3. Courtney TK, Matz S, Webster BS. Disabling occupational injury in the US construction industry, 1996. J Occup Environ Med 2002;44:1161-8.

4. Kovacs FM, Urrutia G, Alarcon JD. Surgery versus conservative treatment for symptomatic lumbar spinal stenosis: a systematic review of randomized controlled trials. Spine (Phila Pa 1976) 2011;36:E1335-51.

5. Dantas FL, Prandini MN, Ferreira MA. Comparison between posterior lumbar fusion with pedicle screws and posterior lumbar interbody fusion with pedicle screws in adult spondylolisthesis. Arq Neuropsiquiatr 2007;65:764-70.

6. Cheng L, Nie L, Zhang L. Posterior lumbar interbody fusion versus posterolateral fusion in spondylolisthesis: a prospective controlled study in the Han nationality. Int Orthop 2009;33:1043-7.

7. Solmaz B, Aydin AL, Gomleksiz C, et al. Skipping posterior dynamic transpedicular stabilization for distant segment degenerative disease. Adv Orthop 2012;2012:496817.

8. Dehoux E, Fourati E, Madi K, Reddy B, Segal P. Posterolateral versus interbody fusion in isthmic spondylolisthesis: functional results in 52 cases with a minimum follow-up of 6 years. Acta Orthop Belg 2004;70:578-82.

9. Fogel GR, Toohey JS, Neidre A, Brantigan JW. Fusion assessment of posterior lumbar interbody fusion using radiolucent cages: X-ray films and helical computed tomography scans compared with surgical exploration of fusion. Spine J 2008;8:570-7.

10. Kambin P. Management of discogenic pain and spinal instability using minimal invasive surgical techniques. In: Kambin P, editor. Arthroscopic and endoscopic spinal surgery text and atlas. 2nd ed. Totowa:
Humana Press; 2005. p.124-5.

11. Cole CD, McCall TD, Schmidt MH, Dailey AT. Comparison of low back fusion techniques: transforaminal lumbar interbody fusion (TLIF) or posterior lumbar interbody fusion (PLIF) approaches. Curr Rev Musculoskelet Med 2009;2:118-26.

12. Suratwala SJ, Pinto MR, Gilbert TJ, Winter RB, Wroblewski JM. Functional and radiological outcomes of 360 degrees fusion of three or more motion levels in the lumbar spine for degenerative disc disease. Spine (Phila Pa 1976) 2009;34:E351-8.

13. Palepu V, Kodigudla M, Goel VK. Biomechanics of disc degeneration. Adv Orthop 2012;2012:726210.

14. Umehara S, Zindrick MR, Patwardhan AG, et al. The biomechanical effect of postoperative hypolordosis in instrumented lumbar fusion on instrumented and adjacent spinal segments. Spine (Phila Pa 1976) 2000;25:1617-24.

15. Antoniou J, Steffen T, Nelson F, et al. The human lumbar intervertebral disc: evidence for changes in the biosynthesis and denaturation of the extracellular matrix with growth, maturation, ageing, and degeneration. J Clin Invest 1996;98:996-1003.

16. White A, Panjabi MM. Clinical biomechanics of the spine. Philedelphia: Lippincott Co.; 1978.

17. Frymoyer JW, Selby DK. Segmental instability. Rationale for treatment. Spine (Phila Pa 1976) 1985;10:280-6.

18. Chen CS, Cheng CK, Liu CL. A biomechanical comparison of posterolateral fusion and posterior fusion in the lumbar spine. J Spinal Disord Tech 2002;15:5363.

19. Wu Y, Tang H, Li Z, Zhang Q, Shi Z. Outcome of posterior lumbar interbody fusion versus posterolateral fusion in lumbar degenerative disease. J Clin Neurosci 2011;18:780-3.

20. Audat Z, Moutasem O, Yousef K, Mohammad B. Comparison of clinical and radiological results of posterolateral fusion, posterior lumbar interbody fusion and transforaminal lumbar interbody fusion techniques in the treatment of degenerative lumbar spine. Singapore Med J 2012;53:183-7.

21. Gioia G, Scotti C, Mandelli D, Sala G. Posterior spinal instrumentation: biomechanical study on the role of rods on hardware response to axial load. Eur Spine J 2011;20 Suppl 1:S3-7.

22. Sanders R, Garcia J, Sheldon GF, Schooley J, Fuchs R, 
Carpenter G. Erythropoietin elevation in anemia of thermal injury. Surg Forum 1976;27:71-2.

23. Xiao Z, Wang L, Gong H, Zhu D. Biomechanical evaluation of three surgical scenarios of posterior lumbar interbody fusion by finite element analysis. Biomed Eng Online 2012;11:31.

24. Hagg O, Fritzell P, Ekselius L, Nordwall A, Swedish Lumbar Spine S. Predictors of outcome in fusion surgery for chronic low back pain. A report from the Swedish Lumbar Spine Study. Eur Spine J 2003;12:2233.

25. Kambin P. Expandable intervertebral cage and surgical method. US Patent 5665122. 1997 Sep 9.

26. Weatherley CR, Prickett CF, O’Brien JP. Discogenic pain persisting despite solid posterior fusion. J Bone
Joint Surg Br 1986;68:142-3.

27. Rivet DJ, Jeck D, Brennan J, Epstein A, Lauryssen C. Clinical outcomes and complications associated with pedicle screw fixation-augmented lumbar interbody fusion. J Neurosurg Spine 2004;1:261-6.

28. Weinstein J, Claverie W, Gibson S. The pain of discography. Spine (Phila Pa 1976) 1988;13:1344-8.

29. Saal JS, Franson RC, Dobrow R, Saal JA, White AH, Goldthwaite N. High levels of inflammatory phospholipase A2 activity in lumbar disc herniations. Spine (Phila Pa 1976) 1990;15:674-8.

30. Lawrence BD, Wang J, Arnold PM, Hermsmeyer J, Norvell DC, Brodke DS. Predicting the risk of adjacent segment pathology after lumbar fusion: a systematic review. Spine (Phila Pa 1976) 2012;37:S123-32. 